\title{
Beamforming correction for dipole measurement using two-dimensional microphone arrays
}

\author{
Yu Liu, ${ }^{\text {a) }}$ Alexander R. Quayle, and Ann P. Dowling \\ Department of Engineering, University of Cambridge, Cambridge CB2 1PZ, United Kingdom \\ Pieter Sijtsma \\ Department of Helicopters and Aeroacoustics, National Aerospace Laboratory NLR, 8300 AD Emmeloord, \\ The Netherlands
}

(Received 8 October 2007; revised 18 April 2008; accepted 19 April 2008)

\begin{abstract}
In this paper, a beamforming correction for identifying dipole sources by means of phased microphone array measurements is presented and implemented numerically and experimentally. Conventional beamforming techniques, which are developed for monopole sources, can lead to significant errors when applied to reconstruct dipole sources. A previous correction technique to microphone signals is extended to account for both source location and source power for two-dimensional microphone arrays. The new dipole-beamforming algorithm is developed by modifying the basic source definition used for beamforming. This technique improves the previous signal correction method and yields a beamformer applicable to sources which are suspected to be dipole in nature. Numerical simulations are performed, which validate the capability of this beamformer to recover ideal dipole sources. The beamforming correction is applied to the identification of realistic aeolian-tone dipoles and shows an improvement of array performance on estimating dipole source powers.
\end{abstract}

(C) 2008 Acoustical Society of America. [DOI: 10.1121/1.2931950]

PACS number(s): 43.38.Hz, 43.28.Tc, 43.25.Zx, 43.28.Ra [AJZ] Pages: 182-191

\section{INTRODUCTION}

Over the past decade, an increasing need has emerged for noise source localization in acoustic measurement. Phased microphone arrays, based on the technique of acoustic beamforming, ${ }^{1,2}$ have been widely applied to experimental acoustics and numerous engineering fields. The theoretical foundation of microphone array was established by Billingsley and Kinns, ${ }^{3}$ who constructed a one-dimensional linear microphone array for real-time jet-noise source location on full-size jet engines. Fisher et al. ${ }^{4}$ also measured jet noise with two microphones using a polar correlation technique. More recent applications of microphone arrays in aeroacoustics include airframe noise, ${ }^{5-7}$ aircraft flyover noise, ${ }^{8-10}$ and ground vehicle noise. ${ }^{11,12}$

The principle idea for microphone array processing techniques is to sum the signals coherently at different microphones to enhance the signal emanating from a focal position while minimizing the contribution from out-of-focus locations. ${ }^{1}$ A crucial step for this technique is to specify a rule, which is referred to as "beamforming," for appropriate summation of the microphone signals to reconstruct the spatial distribution of noise sources. ${ }^{13}$ For the simplest delayand-sum beamformer, the outputs of time-delayed signals are summed, with the delays as a function of focus position and microphone location, to estimate the source distribution. ${ }^{1}$ When a source is at the focus position, the signals add to produce an enhanced signal, whereas for out-of-focus positions, the signals cancel.

\footnotetext{
${ }^{a)}$ Electronic mail: y1275@cam.ac.uk
}

The conventional beamforming algorithm normally assumes monopole propagation characteristics to steer the focus of the array. Although such beamforming techniques work well in locating monopolelike sources with uniform directivities, they can perform poorly when used to reconstruct directional noise sources, such as dipoles. In fact, for many aeroacoustic systems, the noise sources arise from fluctuating loading forces and these are principally of dipole type. Therefore, array measurements can be misinterpreted if applied directly to aeroacoustic sources without considering the source mechanism, particularly if the axes of the dominant dipoles are not in line with the receiver.

Jordan et al. ${ }^{14}$ demonstrated how the monopole assumption can be problematic for a dipole source. They developed a correction for the phase difference in microphone signals to be compatible with a dipole source, and applied it to measurements of a 30 channel linear array for an aeolian-tone dipole produced by cross flow over a cylinder. The true source location and source energy of the dipole was then retrieved. Liu et al. ${ }^{15}$ pointed out the inability of conventional beamforming to directly validate theoretical predictions for dipole-type surface roughness noise. Instead of altering the beamforming algorithm, they processed numerical simulation through the same algorithm as the experiment and compared the simulated and measured source maps. Quayle et al. ${ }^{16}$ have also suggested that a similar mismatch could help explain differences in estimates of source power from two nested arrays. More accurate estimates of aeroacoustic dipoles through array measurements are therefore useful as they can provide insight into the noise generation mecha- 
nism, enable the validation of theoretical and numerical models, and assist the development of noise reduction technologies.

The analysis made by Jordan et al. ${ }^{14}$ suggests a technique for dipole correction of microphone array systems which extend the conventional delay-and-sum procedure to a process of delay-analyze-and-sum. The analysis stage adds an examination of the phase characteristics of microphone signals for each focus position and so is recognized as a "signal correction." The examination process is not very time consuming for a linear focus region, but it will increase the CPU time quadratically in the case of a two-dimensional (2D) focus region, typically with many more grid points. Furthermore, modeling the exact phase alignment of a real acoustic source is difficult due to the inherent nonideal nature of propagation characteristics. A significant drawback of the signal correction is that when extended to a $2 \mathrm{D}$ microphone array it is only applicable to one single dipole with position and direction known. This technique therefore limits the main objective of a phased array, which is to localize noise sources.

In this paper, a beamforming correction to array processing techniques is presented for identifying dipole sources. The main idea is to modify the conventional beamforming algorithm source description to account for the dipole propagation characteristics. This improves the ability of conventional beamforming for estimating dipole sources and extends the technique of Jordan et al., ${ }^{14}$ yielding a new beamforming algorithm capable of evaluating the true source location and source power of multiple dipoles with known orientation. This dipole-beamforming algorithm is then applied to numerical simulations and experiments for validation.

The paper commences with a theoretical formulation for the far-field sound field of aeroacoustic sources in Sec. II. The monopole description is based on Sijtsma ${ }^{17}$ to be consistent with the array software used in this work, and the dipole characteristic term is derived. In Sec. III, two approaches for dipole correction to conventional beamforming techniques, i.e., through microphone signals and beamforming algorithm, are described and compared. A dipolebeamforming algorithm is presented by applying the dipole characteristic term to the source definition for correction. Section IV validates these two approaches through numerical simulations and demonstrates the advantages of the beamforming correction. Following the validation, some results from the application of the beamforming correction to dipole source localization of aeolian tones are presented and discussed in Sec. V.

\section{THEORETICAL FORMULATION}

\section{A. The monopole source}

An ideal point source with uniform directivity (monopole) is assumed to be located at $\boldsymbol{\xi}$ in a medium with a uniform flow $\boldsymbol{U}$. The acoustic pressure $p(\boldsymbol{x}, t)$ at the receiver $\boldsymbol{x}$ satisfies the following convective wave equation: ${ }^{17}$

$$
\frac{1}{c^{2}}\left(\frac{\partial}{\partial t}+\boldsymbol{U} \cdot \nabla\right)^{2} p(\boldsymbol{x}, t)-\nabla^{2} p(\boldsymbol{x}, t)=-Q(t) \delta(\boldsymbol{x}-\boldsymbol{\xi}),
$$

where $c$ is the speed of sound in free field, $Q(t)$ is the monopole strength, and $\delta(\boldsymbol{x}-\boldsymbol{\xi})$ is the Dirac delta function. In the frequency domain, Eq. (1) transforms into the convective Helmholtz equation,

$$
\frac{1}{c^{2}}(i \omega+\boldsymbol{U} \cdot \nabla)^{2} p(\boldsymbol{x}, \omega)-\nabla^{2} p(\boldsymbol{x}, \omega)=-a(\omega) \delta(\boldsymbol{x}-\boldsymbol{\xi}),
$$

where $a(\omega)$ is the Fourier transform of $Q(t)$.

The solution to Eq. (1) is

$$
p(\boldsymbol{x}, t)=\frac{-Q\left(t-\Delta t_{e}\right)}{4 \pi \sqrt{(\boldsymbol{M} \cdot \boldsymbol{r})^{2}+\beta^{2}|\boldsymbol{r}|^{2}}},
$$

where $\boldsymbol{M}=\boldsymbol{U} / \boldsymbol{c}$ is a vector of mean flow Mach number, $\boldsymbol{r}$ $=\boldsymbol{x}-\boldsymbol{\xi}$ is the propagation vector from source to receiver, $\Delta t_{e}$ is the emission time delay,

$$
\Delta t_{e}=\frac{1}{c \beta^{2}}\left(-\boldsymbol{M} \cdot \boldsymbol{r}+\sqrt{(\boldsymbol{M} \cdot \boldsymbol{r})^{2}+\beta^{2}|\boldsymbol{r}|^{2}}\right),
$$

and $\beta^{2}=1-|\boldsymbol{M}|^{2}$.

The frequency-domain form of Eq. (3), i.e., the solution of Eq. (2), can be expressed as

$$
p(\boldsymbol{x}, \omega)=\frac{-a(\omega) e^{-i \omega \Delta t_{e}}}{4 \pi \sqrt{(\boldsymbol{M} \cdot \boldsymbol{r})^{2}+\beta^{2}|\boldsymbol{r}|^{2}}}
$$

because

$$
\begin{aligned}
\int_{-\infty}^{\infty} & p(\boldsymbol{x}, t) e^{-i \omega t} \mathrm{~d} t \\
= & \frac{e^{-i \omega \Delta t_{e}} \int_{-\infty}^{\infty}-Q\left(t-\Delta t_{e}\right) e^{-i \omega\left(t-\Delta t_{e}\right)} \mathrm{d}\left(t-\Delta t_{e}\right)}{4 \pi \sqrt{(\boldsymbol{M} \cdot \boldsymbol{r})^{2}+\beta^{2}|\boldsymbol{r}|^{2}}} .
\end{aligned}
$$

\section{B. The dipole source}

A dipole source can be modeled as a coherent pair of closely placed monopoles $Q(t)$ with opposite phase at a distance $\boldsymbol{l}$ apart. The total source strength is

$$
\boldsymbol{F}_{\text {tot }}=-Q(t)[\delta(\xi)-\delta(\xi-l)] .
$$

If the distance $l=|l|$ is small (i.e., $k l<1$ ), the total source strength simplifies to

$$
\boldsymbol{F}_{\text {tot }}=-\nabla \cdot[\boldsymbol{F}(t) \delta(\xi)]
$$

by the Taylor expansion, where $\boldsymbol{F}(t)=Q(t) \boldsymbol{l}$ is the dipole strength vector. Hence, for a dipole located in a medium with a uniform flow, the convective wave equation [Eq. (1)] is

$$
\frac{1}{c^{2}}\left(\frac{\partial}{\partial t}+\boldsymbol{U} \cdot \nabla\right)^{2} p(\boldsymbol{x}, t)-\nabla^{2} p(\boldsymbol{x}, t)=-\nabla \cdot[\boldsymbol{F}(t) \delta(\boldsymbol{r})],
$$

and the convective Helmholtz equation in the frequency domain is 


$$
\frac{1}{c^{2}}(i \omega+\boldsymbol{U} \cdot \nabla)^{2} p(\boldsymbol{x}, \omega)-\nabla^{2} p(\boldsymbol{x}, \omega)=-a(\omega) \boldsymbol{l} \delta(\boldsymbol{r}) .
$$

Similar to the solution to Eq. (1) for a monopole, the solution of Eq. (9) for source $-\nabla \cdot[\boldsymbol{F}(t) \delta(\boldsymbol{r})]$ is

$$
p(\boldsymbol{x}, t)=-\nabla \cdot\left[\frac{\boldsymbol{F}\left(t-\Delta t_{e}\right)}{4 \pi \sqrt{(\boldsymbol{M} \cdot \boldsymbol{r})^{2}+\beta^{2}|\boldsymbol{r}|^{2}}}\right] .
$$

For a given Mach number vector $\boldsymbol{M}$ and dipole source vector $\boldsymbol{F}$, we can express the solution to Eq. (9) as

$$
\begin{aligned}
p(\boldsymbol{x}, t) & =-\nabla \cdot\left[\frac{\boldsymbol{F}\left(t-\Delta t_{e}\right)}{4 \pi r \alpha}\right] \\
& =\frac{1}{4 \pi \alpha}\left[\frac{\nabla(r \alpha)}{r^{2} \alpha}+\frac{\nabla\left(\Delta t_{e}\right)}{r} \frac{\partial}{\partial t}\right] \cdot \boldsymbol{F}\left(t-\Delta t_{e}\right),
\end{aligned}
$$

where

$$
\begin{aligned}
& \alpha=\sqrt{(\boldsymbol{M} \cdot \boldsymbol{r} / r)^{2}+\beta^{2}}, \\
& \nabla(r \alpha)=\frac{(\boldsymbol{M} \cdot \boldsymbol{r}) \boldsymbol{M}+\beta^{2} \boldsymbol{r}}{r \alpha}, \\
& \nabla\left(\Delta t_{e}\right)=\frac{-\boldsymbol{M}+\nabla(r \alpha)}{c \beta^{2}} .
\end{aligned}
$$

As we are only interested in the far-field sound, we discard the first term in the bracket, $\nabla(r \alpha) / r^{2} \alpha$, and obtain the farfield acoustic pressure as

$$
p(\boldsymbol{x}, t)=\frac{\nabla\left(\Delta t_{e}\right)}{4 \pi r \alpha} \cdot \frac{\partial}{\partial t} \boldsymbol{F}\left(t-\Delta t_{e}\right) .
$$

The Fourier transform of Eq. (16) is

$$
\begin{aligned}
p(x, \omega) & =\frac{\nabla\left(\Delta t_{e}\right)}{4 \pi r \alpha} \cdot i \omega a(\omega) l e^{-i \omega \Delta t_{e}} \\
& =\frac{a(\omega) e^{-i \omega \Delta t_{e}}}{4 \pi r \alpha}\left[i \omega \boldsymbol{l} \cdot \nabla\left(\Delta t_{e}\right)\right] .
\end{aligned}
$$

Comparing the above expression with the pressure spectrum of a monopole in Eq. (5), we obtain the dipole characteristic term representing the ratio between dipole and monopole fields, as indicated

$$
\mathrm{DPL}=-i \omega \boldsymbol{l} \cdot \nabla\left(\Delta t_{e}\right) .
$$

\section{BEAMFORMING ALGORITHM}

\section{A. Conventional beamforming}

Following the work of Sijtsma, ${ }^{17}$ the array processing software stores the measured pressure amplitude in frequency domain in an $\mathrm{N}$-dimensional vector,

$$
\mathbf{p}=\left[p_{1}(f), \ldots, p_{N}(f)\right],
$$

where $N$ is the number of array microphones. The crosspower matrix $\mathbf{C}$ is introduced by

$$
\mathbf{C}=\frac{1}{2} \overline{\mathbf{p p}^{*}},
$$

where the asterisk denotes a complex conjugate and the overbar denotes an ensemble the average or, in practice, average over the Fourier transforms obtained from discrete time blocks. The assumed source description is put in the "transfer vector" g, i.e., its components $g_{n}$ are the pressure amplitudes at the microphone location of an ideal source with unit strength. For the case of a monopole in a medium with uniform flow, $\mathbf{g}$ can be obtained from Eq. (5) by setting the source strength $a(\omega)=1$, namely,

$$
g_{n}=\frac{-e^{-i \omega \Delta t_{e}}}{4 \pi \sqrt{(\boldsymbol{M} \cdot \boldsymbol{r})^{2}+\beta^{2}|\boldsymbol{r}|^{2}}} .
$$

The purpose of beamforming is to determine the amplitude $a$ of sources at grid points $\boldsymbol{\xi}$. This is done by comparing the measured pressure vector $\mathbf{p}$ with the transfer vector $\mathbf{g}$, for instance, through minimization of

$$
J=\overline{|\mathbf{p}-a \mathbf{g}|^{2}} .
$$

The solution of this minimization problem is

$$
a=\frac{\mathbf{g}^{*} \mathbf{p}}{|\mathbf{g}|^{2}}
$$

and the source autopower is

$$
A=\frac{1}{2} \overline{|a|^{2}}=\frac{1}{2} \overline{\frac{\mathbf{g}^{*} \mathbf{p}}{|\mathbf{g}|^{2}}\left(\frac{\mathbf{g}^{*} \mathbf{p}}{|\mathbf{g}|^{2}}\right)^{*}}=\frac{\mathbf{g}^{*} \mathbf{C g}}{|\mathbf{g}|^{4}} .
$$

Expression (24) is known as "conventional beamforming.",

\section{B. Signal correction}

As mentioned earlier, conventional beamforming usually assumes monopole sources to enable the minimization solution. Here, it is referred to as the monopole-beamforming (M-Beam) algorithm. There are two approaches to correct conventional beamforming techniques for dipole source identification. The first approach is to correct the array microphone signals stored in the cross-power matrix $\mathbf{C}$ before the beamforming procedure, as proposed by Jordan et al. ${ }^{14}$ This is essentially a "signal correction" rather than a correction to the beamforming algorithm. The application of this correction is limited for a 2D microphone array because it is time consuming, not easily processed by computer (due to nonideal phase alignment) and restricted to one single fixed dipole source. Nevertheless, the signal correction method provides a useful validation for the dipole characteristic term which will be used in a corrected beamforming algorithm for dipoles.

If the source strength of a monopole is $a(\omega)=1$, we can model a dipole with two coherent monopoles with the same strength but opposite phase. In this case, the signal correction for the cross-power matrix $\mathbf{C}$ would be 


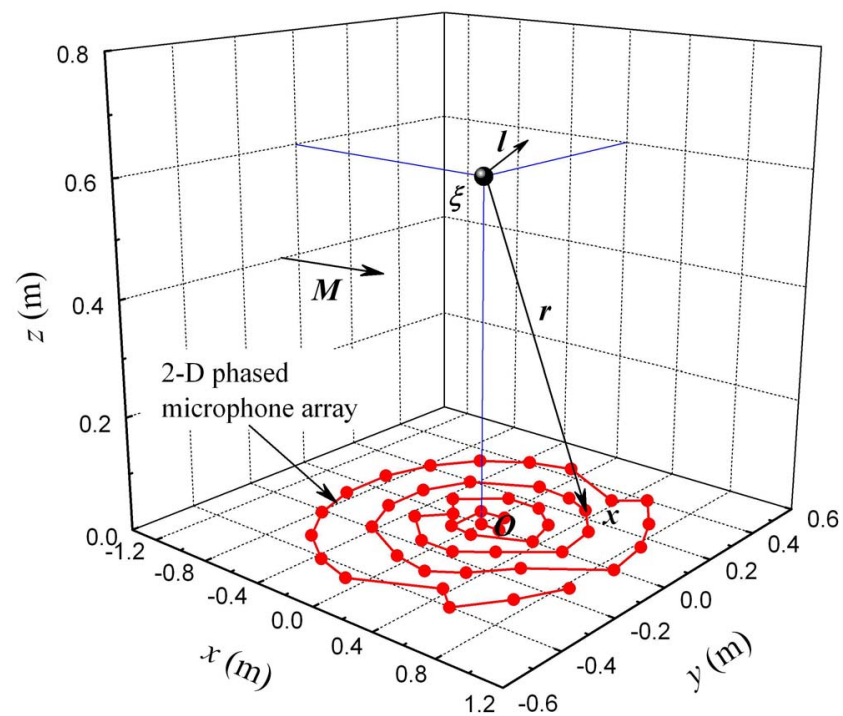

FIG. 1. (Color online) Schematic of the simulation for a dipole source.

$$
C_{m n}=\frac{\frac{1}{2} \overline{p_{m} p_{n}^{*}}}{\mathrm{DPL}_{m} \mathrm{DPL}_{n}^{*}},
$$

where the dipole correction term DPL is an $N$-dimensional vector containing the information of both amplitude and phase for all array microphones, and the suffix denotes the $m$ th or $n$th microphone. If the corrected dipole simulation gives the same source location and source power as the reference monopole simulation, the correction of Eq. (25) is validated.

\section{Beamforming correction}

The second approach for estimating dipole source power is to correct the beamforming algorithm itself to account for a dipole source. It is therefore recognized as the "beamforming correction," and the corrected algorithm is referred to here as the dipole-beamforming (D-Beam) algorithm. To implement the beamforming correction, the transfer vector $\mathbf{g}$ for a dipole should be defined by setting the dipole strength $a(\omega) l=1$ in Eq. (17),

$$
g_{n}=\frac{-e^{-i \omega \Delta t} e_{\text {DPL }}}{4 \pi r \alpha l},
$$

and then the beamforming procedure should proceed as normal to represent the true source location and source power for a dipole. With the dipole signature imprinted in the source description, the beamforming correction allows the user to find the true amplitude and location of a suspected dipole.

In principle, it would be possible to determine the most likely orientation and amplitude of dipoles anywhere on the scanning grid. However, for complex source patterns, which might be encountered on an aeroacoustic model, iterations through amplitude and orientation of potential dipoles for each grid point would be computationally expensive. Such a method might also lead to inaccuracies due to insufficient signal-to-noise ratio. For the present method, we assume that

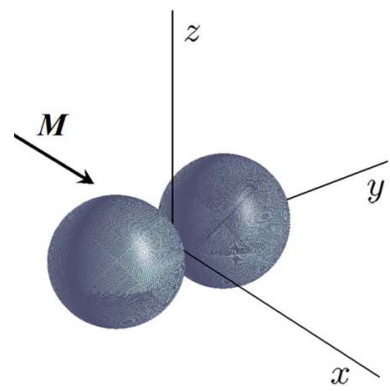

(a)

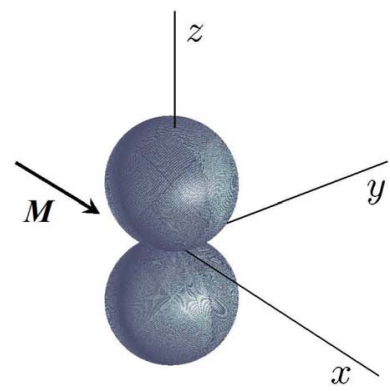

(b)
FIG. 2. (Color online) Dipole directivities for (a) case 1 and (b) case 2 .

the user has some information about likely source orientation and is interested in determining a more accurate estimate of source location and amplitude. A reference dipole direction is therefore required as an input parameter when the D-Beam algorithm is applied. The software divides the region of interest into a number of grid points, and then scans this region point by point for dipoles in the reference direction, estimating the source autopower using Eq. (24).

\section{NUMERICAL SIMULATION}

\section{A. Simulation setup}

In this section, numerical simulations are performed to validate the two approaches mentioned previously. Figure 1 shows a candidate dipole source located in a uniform flow, $\boldsymbol{M}=(0.1,0.0,0.0)$, with the dipole distance vector $\boldsymbol{l}$ at arbitrary directions. In Fig. 2, two cases are examined with the dipole aligned parallel to the $y$ or $z$ axis, referred to as the $Y$ or $Z$ dipole, respectively. The $y-z$ axes are parallel and normal to the array plane, respectively. The source location $\boldsymbol{\xi}$ and dipole vector $\boldsymbol{l}$ for these two test cases are listed in Table I and Figs. 2(a) and 2(b) show the respective dipole directivity patterns. In each test case, an ideal monopole at the same location with source strength $a(\omega)=1$ is also simulated for comparison. As shown in Table I, the dipole size is chosen to be small, $l=0.002 \mathrm{~m}$, to ensure a compact source. Since there is no sound radiation $(\mathrm{DPL}=0)$ in the plane normal to a dipole, an offset of source location $\Delta \xi=0.005 \mathrm{~m}$ is included in case 1 to avoid divide-by-zero errors when applying the dipole correction of Eq. (25).

The simulation array geometry is identical to the nested microphone arrays installed in the Cambridge Markham wind tunnel. ${ }^{16,18}$ The use of both a high-frequency (HF) array and a low-frequency (LF) array allows estimates over large frequency ranges. However, differences in estimates of source power for the same model at common frequencies can be a problem if the sources are not well modeled. Hence, for

TABLE I. Source location $\boldsymbol{\xi}$ and dipole vector $\boldsymbol{l}$ for test cases 1 and 2.

\begin{tabular}{cccc}
\hline \hline Case No. & Dipole & $\boldsymbol{\xi}(\mathrm{m})^{\mathrm{a}}$ & $\boldsymbol{l}(\mathrm{m})^{\mathrm{b}}$ \\
\hline 1 & $Y$ & $(0.0, \Delta \xi, 0.6)$ & $(0.0, \Delta l, 0.0)$ \\
2 & $Z$ & $(0.0,0.0,0.6)$ & $(0.0,0.0, \Delta l)$ \\
\hline \hline
\end{tabular}

$\Delta \xi=0.005 \mathrm{~m}$

$\Delta l=0.002 \mathrm{~m}$. 

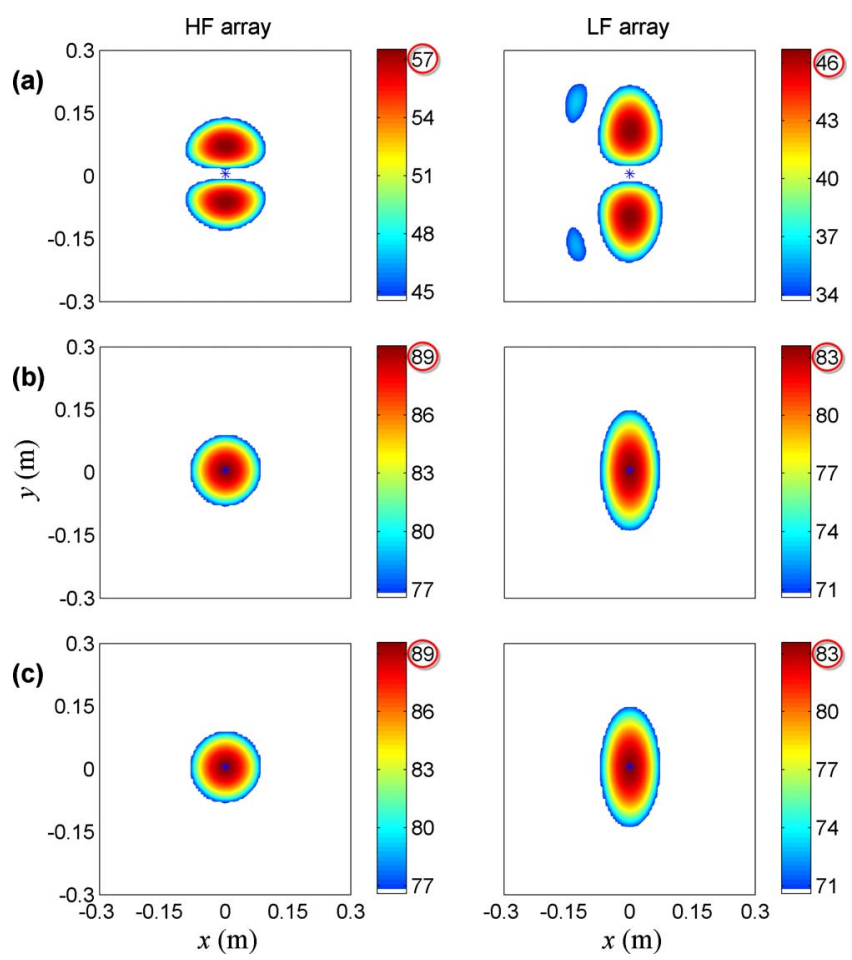

FIG. 3. (Color online) Simulated source maps for case 1: (a) dipole without correction, (b) dipole with signal correction, and (c) reference monopole. HF array, $f=8000 \mathrm{~Hz}$; LF array, $f=2000 \mathrm{~Hz}$.

both simulation and experiment, we look at information from both arrays. The beamforming source maps are generated in $1 / 3$ octave-band frequencies, and the source autopowers have been converted to sound pressure levels [SPL in $\mathrm{dB}$ $\left.=20 \log _{10}\left(p / p_{\text {ref }}\right), p_{\text {ref }}=2 \times 10^{-5} \mathrm{~Pa}\right]$ at a reference distance of $1 / \sqrt{4 \pi} \mathrm{m}$ from the source ${ }^{17}$ and in the dipole direction if the D-Beam algorithm is applied.

\section{B. Signal correction}

Figures 3 and 4 show the simulated source maps for cases 1 and 2 using the signal correction technique. The top row of the figures shows the dipole as interpreted by conventional beamforming (M-Beam algorithm), the middle row is the dipole after signal correction, and the bottom row gives the reference monopole with conventional beamforming. For brevity, only the results of 8000 and $2000 \mathrm{~Hz}$ are shown for the HF and LF arrays, respectively.

For the $Y$ dipole, Fig. 3 shows that the dipole source is missed at the true location (marked by an asterisk) by the conventional beamforming because the phase variation in the radiated sound is entirely different from a monopole in directions close to the normal of the dipole vector $\boldsymbol{l}$, i.e., the sign of the acoustic pressure changes across this normal plane. Instead, the sound is interpreted as coming from elsewhere on the scan grid. More striking are the dipole source powers that are significantly underestimated compared to the reference monopole simulations. As can be found from the color bars in Fig. 3, without correction, the detected peak source powers are 57.6 and $46.7 \mathrm{~dB}$ for the HF and LF arrays, respectively, which are about 32 and $37 \mathrm{~dB}$ lower than those of the reference monopoles used to construct the dipoles.
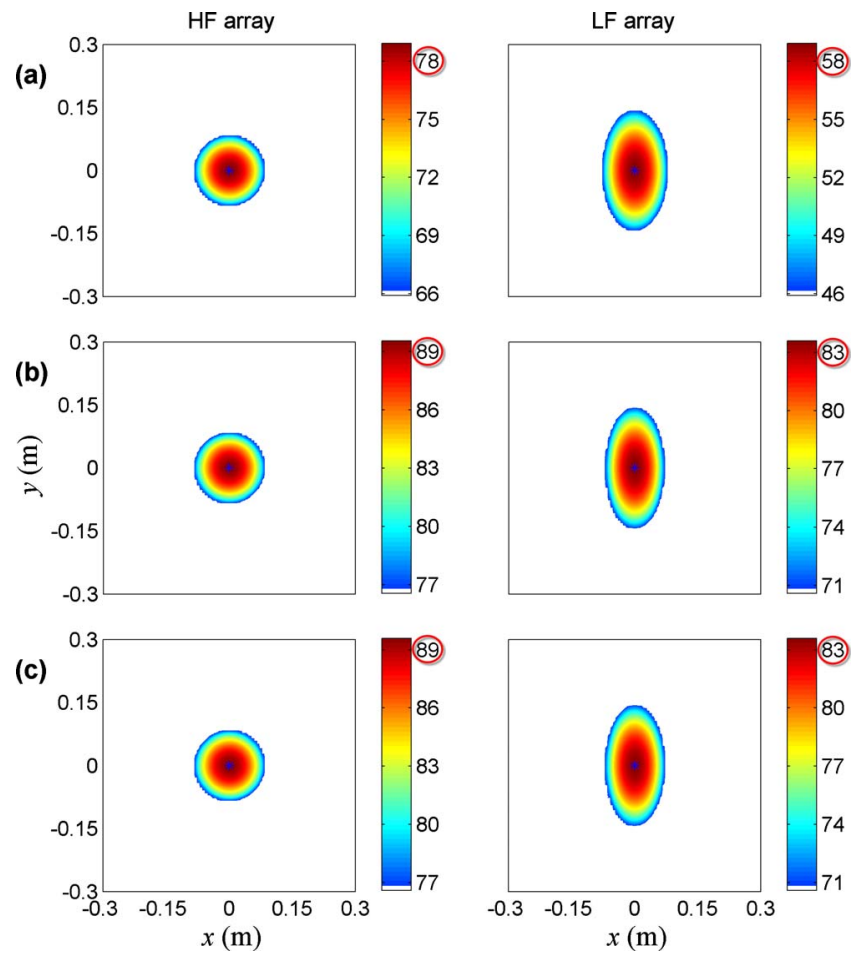

FIG. 4. (Color online) Simulated source maps for case 2: (a) dipole without correction, (b) dipole with signal correction, and (c) reference monopole. HF array, $f=8000 \mathrm{~Hz}$; LF array, $f=2000 \mathrm{~Hz}$.

Conventional beamforming is able to better detect the source at the original dipole location for case 2 (Fig. 4), where the radiation of the $Z$ dipole has a phase variation similar to that of a monopole over the area of the arrays. However, the source powers before correction are still much lower than those of the reference monopole, which can be largely accounted for by the relatively small value of the term DPL in the dipole pressure spectrum [Eq. (17)]. The frequency-dependent DPL also explains the $20 \mathrm{~dB}$ difference between the source powers (without correction) detected by the HF and LF arrays because of the different chosen frequencies, 8000 and $2000 \mathrm{~Hz}$, respectively.

With the signal correction applied, however, the true source maps for a dipole are recovered for both cases 1 and 2 , and they agree with those of the reference monopole in both source pattern and source power, as shown clearly in the middle rows and bottom rows of Figs. 3 and 4. This provides confirmation for the validity of the dipole correction form [Eq. (25)].

Figure 5 shows the variation of the peak source power $S$ with $1 / 3$ octave-band center frequency $f$ for cases 1 and 2 with both arrays. For a monopole with $a(\omega)$ independent of frequency, $S$ scales on $f$ because the $1 / 3$-octave bandwidth $B$ varies linearly with $f$. Actually, $S$ should increase by $1 \mathrm{~dB}$ between two adjacent $1 / 3$ octave-band center frequencies $f_{1}$ and $f_{2}$ because

$$
\begin{aligned}
\Delta S & =10 \log _{10}\left(B_{2} / B_{1}\right)=10 \log _{10}\left(f_{2} / f_{1}\right) \\
& =10 \log _{10} 2^{1 / 3}=1 \mathrm{~dB},
\end{aligned}
$$

and the corrected estimates (solid lines) show this tendency. However, without correction, estimates of $S$ (solid squares and circles) vary with $f^{3}$ in each case because the sound 

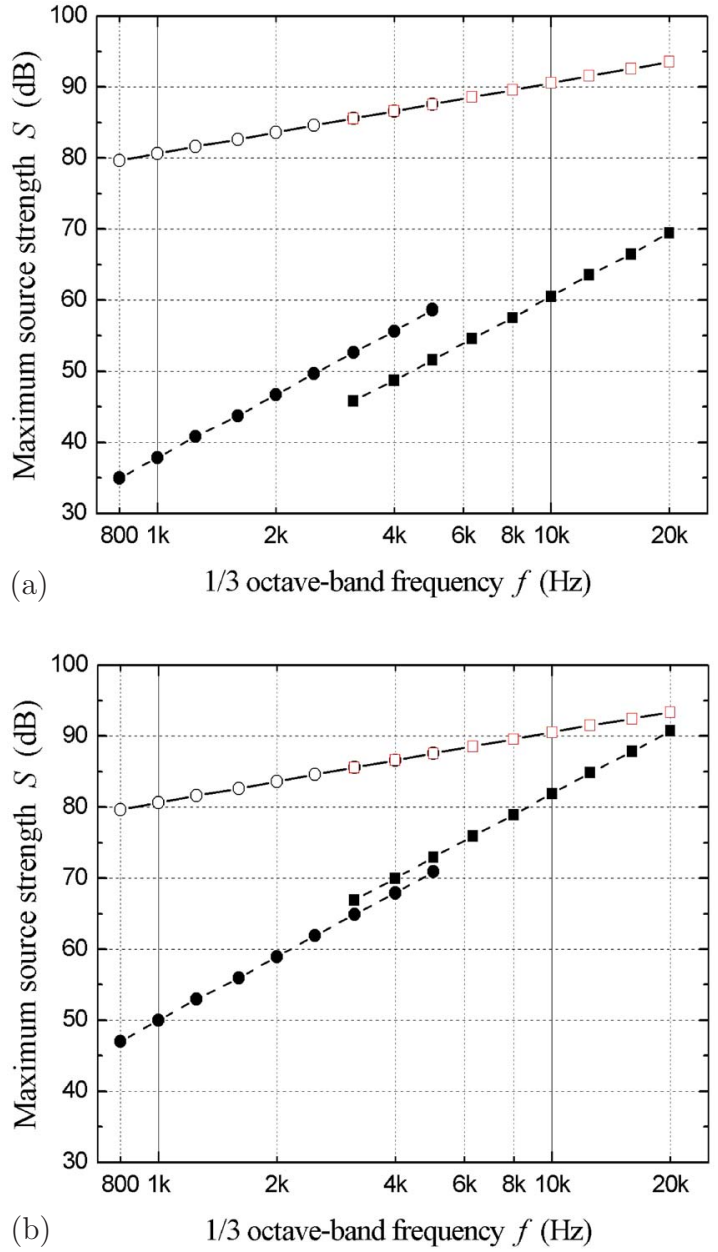

FIG. 5. (Color online) Variation of peak source power $S$ with $1 / 3$ octaveband center frequency $f$ for (a) case 1 and (b) case 2. Dipole without cor-

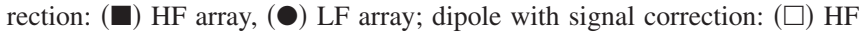
array, (○) LF array; --- $S \sim f^{3},-S \sim f$.

power of a dipole scales on $\omega^{2}$ as well as the frequencydependent $1 / 3$-octave bandwidth $B$, so that

$$
\begin{aligned}
\Delta S & =10 \log _{10}\left(f_{2}^{2} B_{2} / f_{1}^{2} B_{1}\right)=10 \log _{10}\left(f_{2}^{3} / f_{1}^{3}\right) \\
& =10 \log _{10} 2=3 \mathrm{~dB} .
\end{aligned}
$$

After correction, estimates of $S$ (open squares and circles) vary with frequency as $S \sim f$, which is simply a $1 / 3$-octave bandwidth dependence. The same relationship is seen for estimates of a monopole with uniform strength. The signal correction also presents the same values of $S$ at a fixed frequency for the HF and LF arrays, which is in line with the expectation that the algorithm should be independent of the array geometry.

TABLE II. Simulated peak source powers $S(\mathrm{~dB})$ by the M-Beam and D-Beam algorithms for cases 1 and 2 . HF array, $f=8000 \mathrm{~Hz}$; LF array, $f$ $=2000 \mathrm{~Hz}$.

\begin{tabular}{ccccccc}
\hline \hline & & \multicolumn{2}{c}{ HF array } & & \multicolumn{2}{c}{ LF array } \\
\cline { 3 - 4 } \cline { 6 - 7 } Case no. & Dipole & M-Beam & D-Beam & & M-Beam & D-Beam \\
\hline 1 & $Y$ & 57.55 & 79.11 & & 46.71 & 61.10 \\
2 & $Z$ & 78.92 & 79.12 & & 58.93 & 61.12 \\
\hline \hline
\end{tabular}
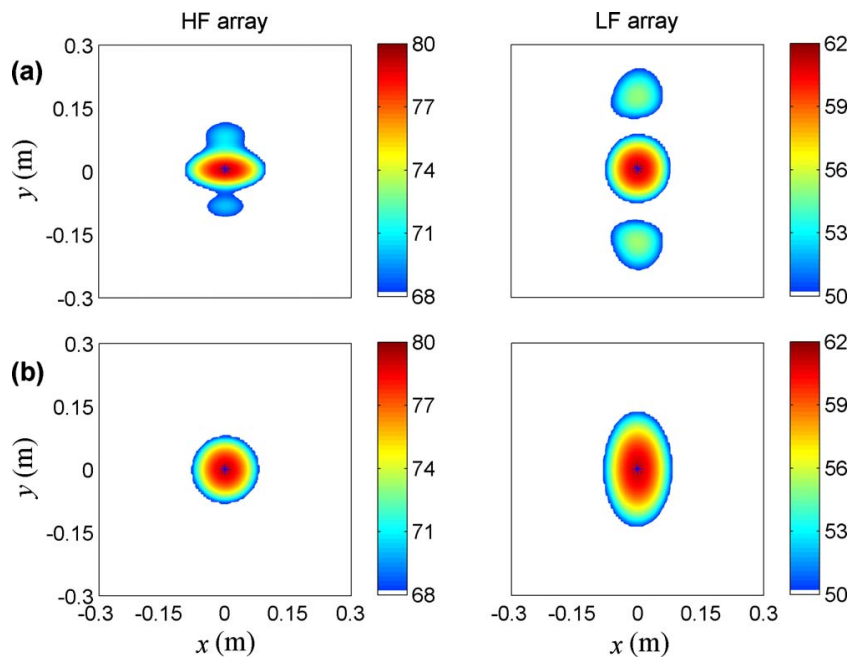

FIG. 6. (Color online) Simulated source maps by the D-Beam algorithm for (a) case 1 and (b) case 2. HF array, $f=8000 \mathrm{~Hz}$; LF array, $f=2000 \mathrm{~Hz}$.

\section{Beamforming correction}

With the dipole correction term DPL validated by the signal correction method, we apply the D-Beam algorithm to identify the original dipole source instead of the corrected monopole source as in the middle rows of Figs. 3 and 4. The effect of the beamforming correction on the source maps of cases 1 and 2 is shown in Fig. 6. We can see from the corrected algorithm that the main source is correctly placed at the true source location, although low-level side lobes occur away from the main source. Note that the microphone arrays now detect very similar peak source powers $S$ for both dipole orientations (see Table II).

Comparing the source maps of the signal correction (Figs. 3 and 4) and beamforming correction (Fig. 6), we see that both techniques are able to recover the true location of a single dipole. However, the signal correction gives the amplitude of the constituent monopoles of the dipole, whereas the beamforming correction directly identifies the amplitude of the dipole. Table III shows the elapsed CPU time of the two techniques for cases 1 and 2 on a personal computer with an Intel Pentium 4 processor of $3.4 \mathrm{GHz}$ clock speed and a 1 Gbyte random access memory. The CPU time is very close in each column of Table III and it is evident that the signal correction uses much longer CPU time than the beamforming correction, i.e., $t_{s} \sim 7 t_{b}$, because the signal correction includes a process of delay-analyze-and-sum while the beamforming correction processes the data directly using the D-Beam algorithm. The CPU time varies significantly between the HF and LF arrays due to the different frequencies

TABLE III. Comparison of elapsed CPU time (s) between the signal correction $\left(t_{s}\right)$ and beamforming correction $\left(t_{b}\right)$. HF array, $f=8000 \mathrm{~Hz}$; LF array, $f=2000 \mathrm{~Hz}$.

\begin{tabular}{cccccccc}
\hline \hline & & \multicolumn{2}{c}{ HF array } & & \multicolumn{2}{c}{ LF array } \\
\cline { 3 - 4 } \cline { 6 - 7 } Case no. & Dipole & $t_{s}$ & $t_{b}$ & & $t_{s}$ & $t_{b}$ \\
\hline 1 & $Y$ & 141.7 & 21.4 & & 41.6 & 6.8 \\
2 & $Z$ & 141.8 & 21.4 & & 41.4 & 6.7 \\
\hline \hline
\end{tabular}

Liu et al.: Beamforming correction for dipole measurement 


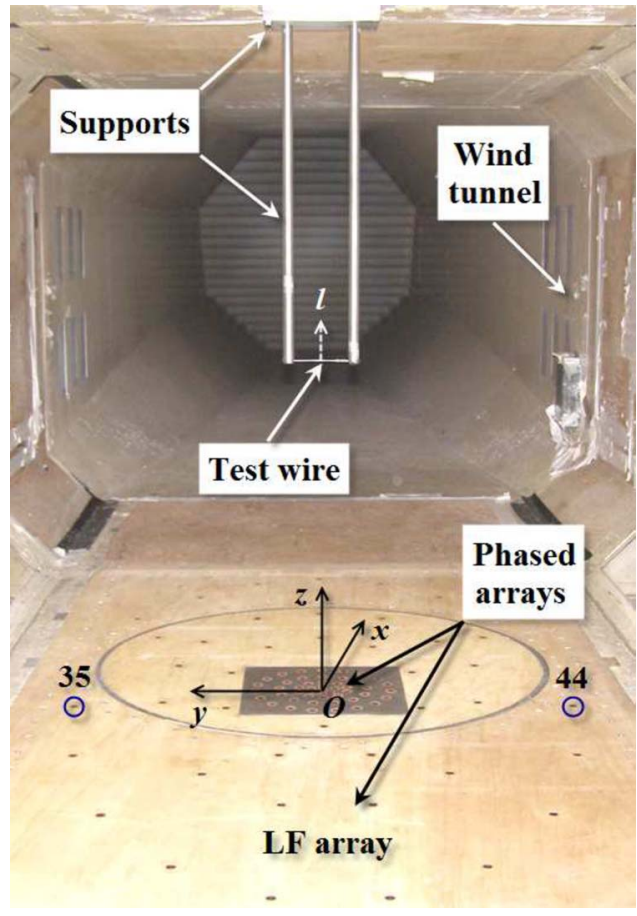

(a)

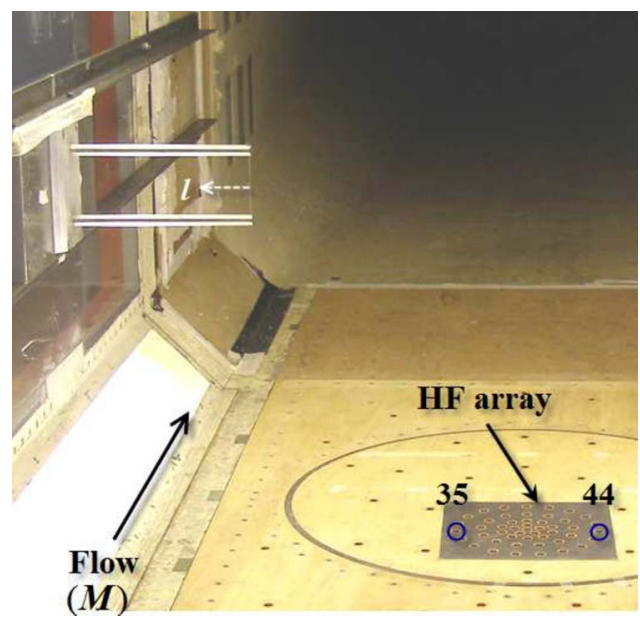

(b)

FIG. 7. (Color online) Experimental setup for aeolian-tone dipoles in two different directions: (a) $Z(0,0,1)$ and (b) $Y(0,1,0)$.

used. With an identical frequency bandwidth for both arrays $(\Delta f=117.2 \mathrm{~Hz}$, see Sec. V A), the higher center frequency of $1 / 3$ octave-band contains more frequency bands and thus takes longer CPU time to process. In addition, the beamforming correction has the advantage over the signal correction in that it can be used in the case of multiple dipoles, as we show in Sec. V.

\section{EXPERIMENT}

\section{A. Experimental setup}

A series of experiments were conducted in the lowspeed Markham wind tunnel at the Department of Engineering, University of Cambridge, to test the performance of the D-Beam algorithm. Figure 7 illustrates the experimental
TABLE IV. Dipoles in two different directions.

\begin{tabular}{ccccc}
\hline \hline & & \multicolumn{3}{c}{ Coordinates of wire center $(\mathrm{m})$} \\
\cline { 3 - 5 } Dipole & Direction & \multicolumn{1}{c}{$x$} & $y$ & $z$ \\
\hline$Z$ & $(0,0,1)$ & 0.075 & 0.003 & 0.60 \\
$Y$ & $(0,1,0)$ & -0.005 & 0.465 & 0.60 \\
\hline \hline
\end{tabular}

setup of the wind tunnel, the phased microphone arrays, and a test wire with supports. The test wire was $1.5 \mathrm{~mm}$ in diameter and aligned perpendicular to the flow in order to generate an aeolian tone, representing a realistic aeroacoustic dipole source. Acoustic measurements were performed at free stream flow velocities, $U=20,40 \mathrm{~m} / \mathrm{s}$, to obtain aeolian-tone dipole sound at different peak frequencies. The wire was orientated to hopefully obtain dipoles in the $Z$ and $Y$ directions, as shown in Table IV. The " $Y$ dipole" configuration was moved to the side to provide a better view for the microphone arrays. The support structure included two support rods $10 \mathrm{~cm}$ apart and one or two support plates, and was mounted to the tunnel ceiling or side wall.

Measurements were made with both the HF and LF arrays at sampling frequency of 120 or $30 \mathrm{kHz}$, respectively. Both arrays contain 48 microphones and are flush mounted into the rigid tunnel floor. In the post processing, the timedomain signals were broken down into 1024 (HF array) or 256 (LF array) data blocks, and each block Fourier transformed, resulting in frequency-domain signals with bandwidth $\Delta f=117.2 \mathrm{~Hz}$ for each array. Narrow-band acoustic beamforming scans were performed to determine source autopowers, as described in Sec. III. Both monopole (M-Beam) and dipole (D-Beam) source descriptions were examined in each case. For each experiment, a horizontal scan plane was used which intersected the test wire at the midpoint. Finally, source maps were generated by summing the narrow-band data to $1 / 3$ octave-band data, with source auto-powers shown as SPL (dB), at a reference distance of $1 / \sqrt{4 \pi} \mathrm{m}$ and a reference dipole direction if applicable.

\section{B. Noise spectra}

Cross spectra between a pair of microphones are shown in Fig. 8 for each experimental case together with the noise generated by the support (without the wire) for comparison. Microphones used for the cross spectra are marked by circles in Figs. 7(a) and 7(b) for each array. The aeolian tones are clearly audible around $6000 \mathrm{~Hz}$ for the HF array $(U$ $=40 \mathrm{~m} / \mathrm{s})$ and $3000 \mathrm{~Hz}$ for the LF array $(U=20 \mathrm{~m} / \mathrm{s})$, corresponding to a Strouhal number of $\sim 0.225$. As the spectral peaks span a range of frequencies, 1/3-octave frequency bands were used for beamforming source maps to capture the total acoustic energy of the aeolian tone. In this case, the center frequencies of $6300 \mathrm{~Hz}$ (HF array) and $3150 \mathrm{~Hz}$ (LF array) were selected because they cover the frequency bands of 5613-7072 and $2806-3536 \mathrm{~Hz}$, respectively, which are sufficiently large for the spectral peaks of interest. 

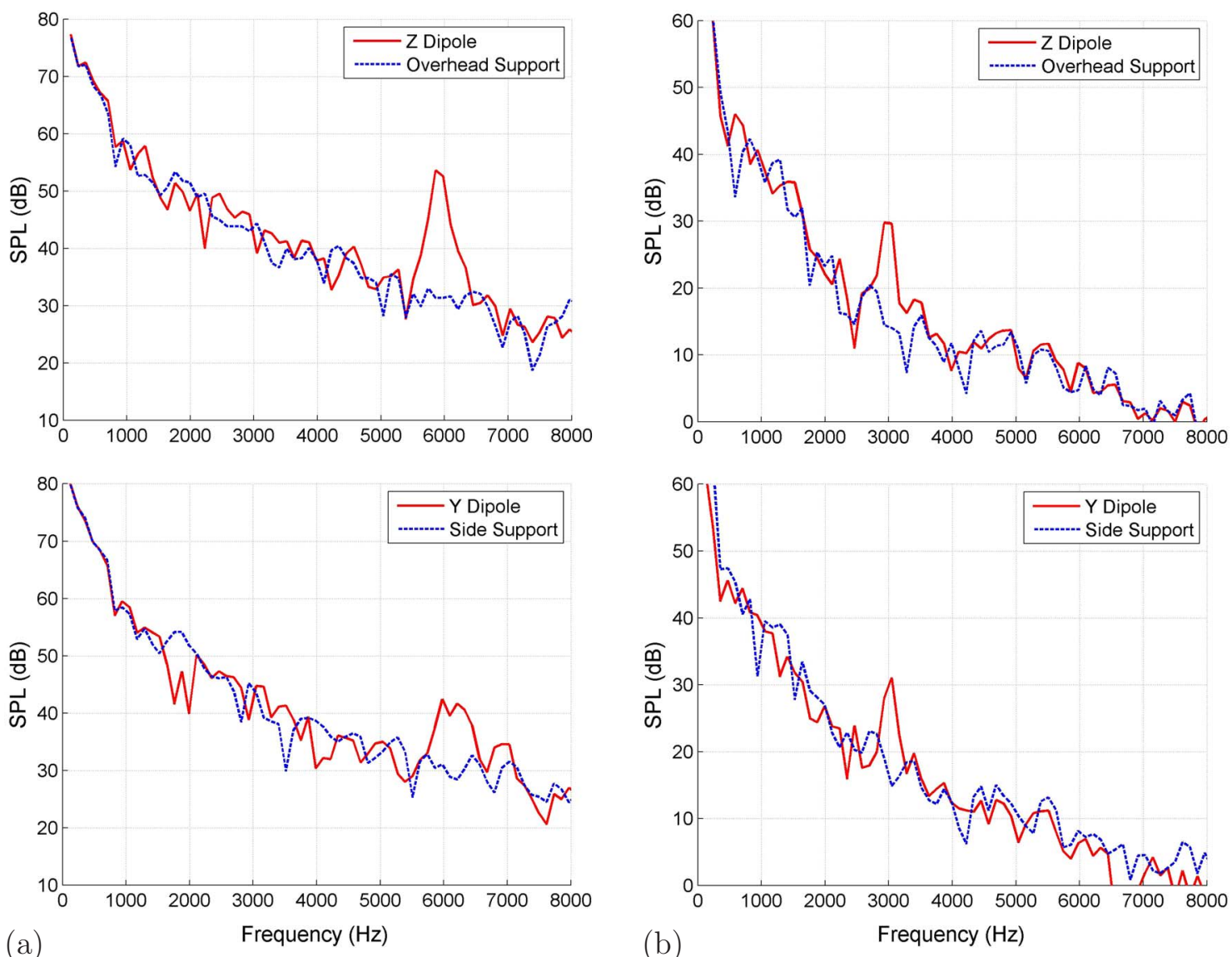

FIG. 8. (Color online) Measured cross spectra for dipoles in two different directions: $Z(0,0,1)$ and $Y(0,1,0)$ with cross spectra of overhead and side supports for comparison. (a) HF array, $U=40 \mathrm{~m} / \mathrm{s}$; (b) LF array, $U=20 \mathrm{~m} / \mathrm{s} ; \Delta f=117.2 \mathrm{~Hz}$.

\section{Beamforming correction}

\section{Simulation}

Theoretical simulations were first carried out to examine the performance of the proposed beamforming correction on identifying distributed aeolian-tone dipoles, and to provide a baseline for comparison with experimental results. Liu et $a l .{ }^{15}$ used this method to validate their prediction model for surface roughness noise by comparing the measured and simulated source powers. In this work, however, we are not concerned about the source power itself. Instead, we compare the increase $\left(\Delta S_{s}\right)$ in the estimated peak source power $S$ from the M-Beam algorithm to the D-Beam algorithm. To implement the simulation for the $Z$ and $Y$ dipoles, we assume that 11 identical incoherent dipoles are uniformly distributed in the direction of the wire, and the same value of $a(\omega)$ is used as the input source strength for both algorithms and both arrays.

Figure 9 illustrates the simulated source maps at $6300 \mathrm{~Hz}$ by the HF array for the $Z$ and $Y$ dipoles, and the comparison between the M-Beam and D-Beam algorithms. The color bar on the right restricts the SPL data within the range of $0-15 \mathrm{~dB}$, and the source maps of different dipoles and algorithms are shown on identical scales for comparison. Support structures are also sketched on the source maps. Note that since $a(\omega)$ has been set equal to some value, these simulated SPL data do not represent the true source powers of the aeolian-tone dipoles and are merely meaningful as relative levels to show the effect of the beamforming correction. In fact, both the M-Beam and D-Beam algorithms detect the true location of the main source for the distributed dipoles. However, only the D-Beam algorithm correctly identifies strength of the $Z$ and $Y$ dipoles, as both dipoles have the similar source powers. The M-Beam algorithm ap-
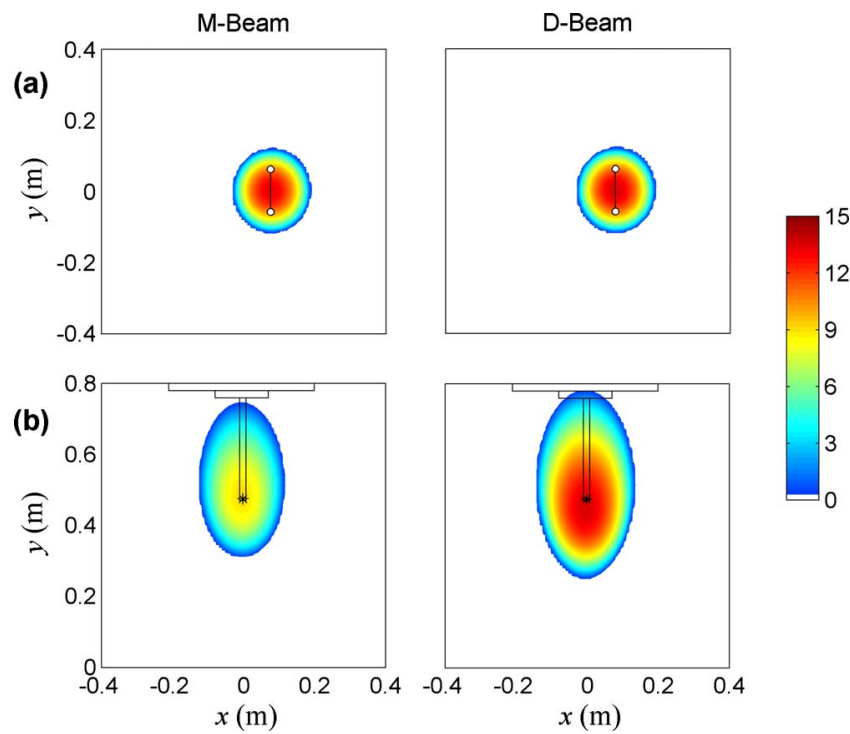

FIG. 9. (Color online) Comparison of simulated source maps by the M-Beam and D-Beam algorithms for dipoles in two different directions: (a) $Z(0,0,1)$ and (b) $Y(0,1,0)$. HF array, $f=6300 \mathrm{~Hz}$. 


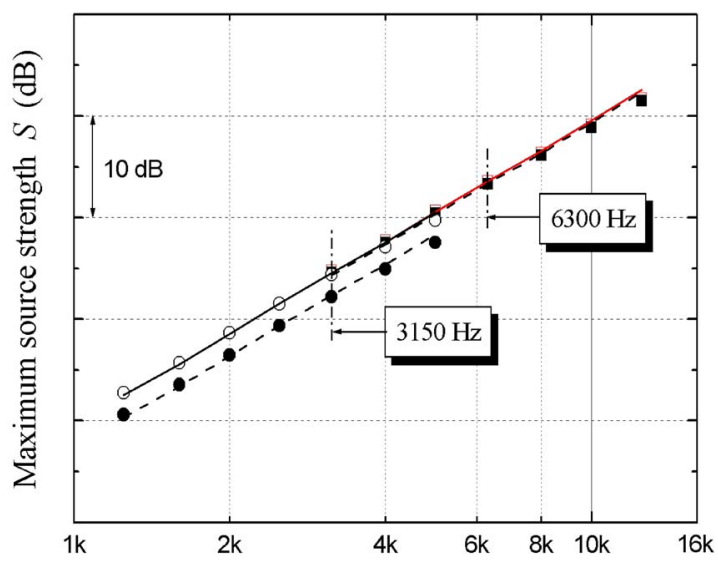

(a)

1/3 octave-band frequency $f(\mathrm{~Hz})$

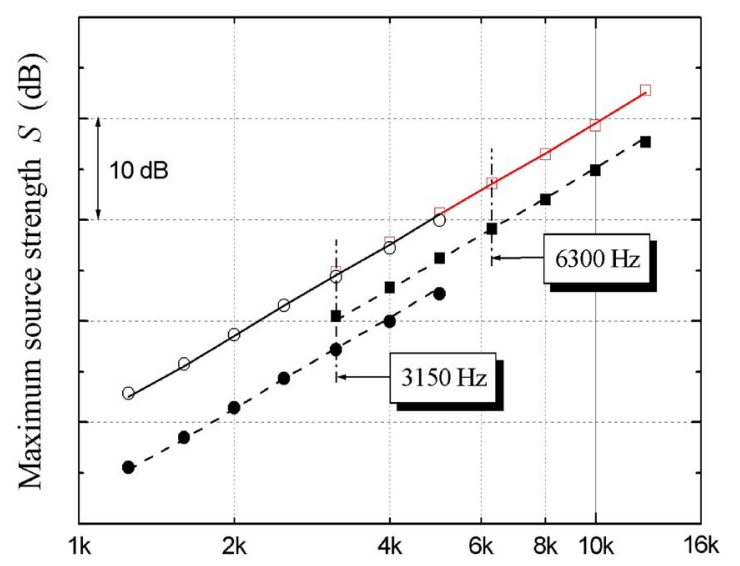

(b)

1/3 octave-band frequency $f(\mathrm{~Hz})$

FIG. 10. (Color online) Variation of simulated peak source power $S$ with $1 / 3$ octave-band center frequency $f$ for (a) $Z$ dipole and (b) $Y$ dipole.

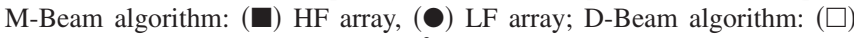
HF array, $(\bigcirc)$ LF array; ---, $-S \sim f^{3}$.

pears to substantially underestimate source strength of the $Y$ dipoles, as anticipated by earlier simulation (Fig. 3).

Figures 10(a) and 10(b) show the variation of $S$ with $1 / 3$ octave-band center frequency $f$. As expected from Eq. (28), the simulated $S$ of the $Z$ and $Y$ dipoles varies as $f^{3}$, independently of the different arrays and algorithms used. After applying the beamforming correction, the estimated peak source powers $S$ by the HF and LF arrays almost coincide with one another, consistent with the tendency of the ideal individual sources as in Fig. 5. For the $Z$ dipole, the effect of
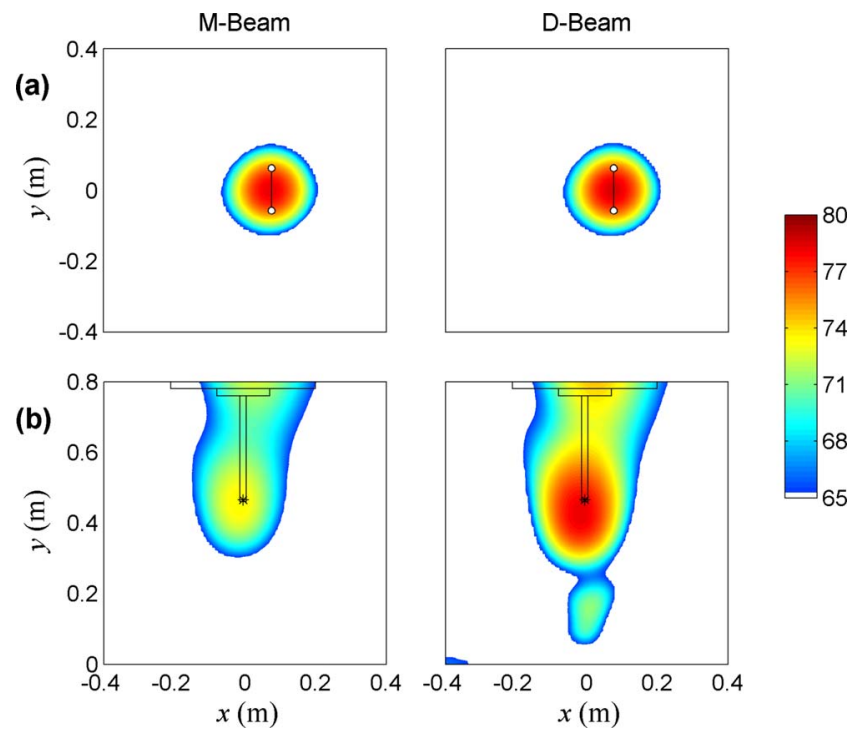

FIG. 11. (Color online) Comparison of measured source maps by the M-Beam and D-Beam algorithms for dipoles in two different directions: (a) $Z(0,0,1)$ and (b) $Y(0,1,0)$. HF array, $f=6300 \mathrm{~Hz}$.

the beamforming correction is generally small, i.e., $\Delta S_{s}$ $\approx 2.2 \mathrm{~dB}$ for the LF array and nearly negligible for the $\mathrm{HF}$ array (see Table V). However for the $Y$ dipole, the values of $S$ increase significantly from the M-Beam algorithm to the D-Beam algorithm by about 7.2 dB (LF array) and $4.6 \mathrm{~dB}$ (HF array). This is because for the $Z$ dipole the variation of phase over the microphones is just due to propagation distance and is correctly captured by the M-Beam algorithm. However, for the $Y$ dipole, there is an additional phase change due to the dipole directivity.

\section{Measurement}

Figure 11 illustrates the measured source maps at $6300 \mathrm{~Hz}$ by the HF array for the $Z$ and $Y$ dipoles. The source powers of the aeolian-tone dipoles identified by the M-Beam and D-Beam algorithms are shown in the SPL range of 65-80 dB. Comparing Figs. 9 and 11, we see that the simulated and measured source maps show very similar source patterns and similar effects of the beamforming correction.

Table V compares the estimated peak source power $S$ of the two dipoles, as recorded by both of the nested arrays at 6300 and $3150 \mathrm{~Hz}$. For each dipole orientation, the value of $S$ is given for both the M-Beam and D-Beam algorithms. In

TABLE V. Peak source powers $S(\mathrm{~dB})$ of measurement and simulation for the $Z$ and $Y$ dipoles. (a) HF array, $U=40 \mathrm{~m} / \mathrm{s}, f=6300 \mathrm{~Hz}$; (b) LF array, $U=20 \mathrm{~m} / \mathrm{s}, f=3150 \mathrm{~Hz}$.

\begin{tabular}{|c|c|c|c|c|c|}
\hline \multirow[b]{2}{*}{ Dipole } & \multicolumn{2}{|c|}{ Measurement } & \multicolumn{2}{|c|}{ Simulation } & \multirow[b]{2}{*}{$\left|\Delta S_{m}-\Delta S_{s}\right|$} \\
\hline & M-Beam & D-Beam & $\Delta S_{m}$ & $\Delta S_{s}$ & \\
\hline \multicolumn{6}{|l|}{ (a) } \\
\hline$Z$ & 78.31 & 78.57 & 0.26 & 0.24 & 0.02 \\
\hline$Y$ & 73.59 & 78.32 & 4.73 & 4.57 & 0.16 \\
\hline \multicolumn{6}{|l|}{ (b) } \\
\hline$Z$ & 51.73 & 53.92 & 2.19 & 2.16 & 0.03 \\
\hline Y & 48.01 & 55.18 & 7.17 & 7.22 & 0.05 \\
\hline
\end{tabular}


each case, the M-Beam algorithm shows a lower estimate of source power. The differences between the M-Beam and D-Beam algorithm are also much greater for the $Y$ dipole, as seen in simulation. In addition, the D-Beam algorithm correctly identifies the wire as producing very similar sound power in both orientations. Corrected source maps are also shown in the right column of Fig. 11.

The difference in the measured peak source powers between the M-Beam and D-Beam algorithms is shown in Table $\mathrm{V}$ as $\Delta S_{m}$. Due to the source directivity, the $Y$ dipole shows larger $\Delta S_{m}$ because much of its sound radiation is missed without the beamforming correction. The corresponding values of $\Delta S_{s}$ obtained from a simplified simulation of the experimental setup are shown in Table V. This enables the evaluation of the difference $\left|\Delta S_{m}-\Delta S_{s}\right|$. It indicates that $\Delta S_{m}$ agrees with $\Delta S_{s}$ fairly well for all cases with the largest discrepancy of $0.16 \mathrm{~dB}$, occurring at the $Y$ dipole by the $\mathrm{HF}$ array. This suggests that the modeling of the aeolian tones from the wire as a series of incoherent dipoles is realistic and provides a preliminary validation of the D-Beam algorithm for an experimental dipole source.

\section{CONCLUSIONS}

It has been shown that conventional beamforming techniques can misinterpret the microphone array measurement for a simple dipole due to the inherent monopole assumption. A correction for the phase differences in microphone signals was presented by Jordan et al. ${ }^{14}$ and it displayed the capability to recover the source location of a single dipole in the resulting source map. In this paper, we present a method of modifying the source transfer vector to search the scanning plane for dipole sources. The resulting algorithm is able to identify the source location and amplitude of dipoles with a suspected orientation. This method extends the capability of the signal correction by Jordan et al. ${ }^{14}$ to deal with multiple dipoles.

In simulations of dipoles normal to the flow, the dipolebeamforming (D-Beam) algorithm recovered the true source location and amplitude. For a point dipole, the use of a dedicated dipole-beamforming algorithm can lead to large increases in source estimates. For simulations with distributed dipole sources and comparable measurements of aeolian tones, the improvement in estimates of source power with the D-Beam algorithm was less marked. However, the implementation of the new algorithm did allow recovery of the same source strength from different orientations of the same aeolian tone.

While both simulations and measurements have validated the capability of the D-Beam algorithm to improve conventional techniques for identifying dipole sources, the present implementation requires specification of the dipole direction. A potential improvement would be to iterate through different dipole orientations at each point on the scanning grid. However, in many aeroacoustic experiments, it may be possible to readily identify the likely dipole orientations by using knowledge of the source mechanisms. In such cases, it is hoped that more accurate estimates of source amplitude can be determined with this method.

\section{ACKNOWLEDGMENTS}

Financial support from the Cambridge-MIT Institute is gratefully acknowledged. The authors would like to thank the National Aerospace Laboratory (NLR), The Netherlands, for assisting in the design and manufacture of the microphone array system and providing the analysis software (Ref. 18). Y. Liu acknowledges the Overseas Research Students Awards Scheme (ORSAS) and the Gates Cambridge Scholarships and A. R. Quayle is grateful to the Engineering and Physical Science Research Council (EPSRC), U.K. for doctoral research funding.

${ }^{1}$ D. H. Johnson and D. E. Dudgeon, Array Signal Processing: Concepts and Techniques (Prentice-Hall, London, 1993).

${ }^{2}$ R. P. Dougherty, "Beamforming in acoustic testing," in Aeroacoustic Measurements, edited by T. J. Mueller (Springer-Verlag, Berlin, 2002).

${ }^{3}$ J. Billingsley and R. Kinns, "The acoustic telescope," J. Sound Vib. 48, 485-510 (1976).

${ }^{4}$ M. J. Fisher, M. Harper-Bourne, and S. A. L. Glegg, "Jet engine noise source location: The polar correlation technique," J. Sound Vib. 51, 23-54 (1977)

${ }^{5}$ J. F. Piet and G. Elias, "Airframe noise source localization using a microphone array," AIAA Paper No. 97-1643, May 1997.

${ }^{6}$ J. A. Hayes, W. C. Horne, P. T. Soderman, and P. H. Brent, "Airframe noise characteristics of a $4.7 \%$ scale DC-10 model," AIAA Paper No. 97-1594, May 1997.

${ }^{7}$ W. H. Herkes and R. W. Stoker, "Wind tunnel measurements of the airframe noise of a high-speed civil transport," AIAA Paper No. 98-0472, January 1998.

${ }^{8}$ U. Michel, B. Barsikow, J. Helbig, M. Hellmig, and M. Schuttpelz, "Flyover noise measurements on landing aircraft with a microphone array," AIAA Paper No. 98-2336, June 1998.

${ }^{9} \mathrm{U}$. Michel and W. Qiao, "Directivity of landing gear noise based on flyover measurements," AIAA Paper No. 99-1956, May 1999.

${ }^{10}$ J. F. Piet, G. Elias, and P. Lebigot, "Localization of acoustic source from a landing aircraft with a microphone array," AIAA Paper No. 99-1811, May 1999.

${ }^{11}$ B. Barsikow, W. F. King, and E. Pfizenmaier, "Wheel/rail noise generated by a high-speed train investigated with a line array of microphones," J. Sound Vib. 118, 99-122 (1987).

${ }^{12}$ H. Kook, G. B. Moebs, P. Davies, and J. S. Bolton, "An efficient procedure for visualizing the sound field radiated by vehicles during standardized passby tests," J. Sound Vib. 233, 137-156 (2000).

${ }^{13}$ S. R. Venkatesh, D. R. Polak, and S. Narayanan, "Beamforming algorithm for distributed source localization and its application to jet noise," AIAA J. 41, 1238-1246 (2003)

${ }^{14}$ P. Jordan, J. A. Fitzpatrick, and J.-C. Valière, "Measurement of an aeroacoustic dipole using a linear microphone array," J. Acoust. Soc. Am. 111, 1267-1273 (2002).

${ }^{15}$ Y. Liu, A. P. Dowling, and H.-C. Shin, "Measurement and simulation of surface roughness noise using phased microphone arrays," J. Sound Vib. 314, 95-112 (2008).

${ }^{16}$ A. R. Quayle, A. P. Dowling, H. Babinsky, W. R. Graham, and Y. Liu, "Phased array measurements from landing gear models," AIAA Paper No. 2007-3463, May 2007.

${ }^{17} \mathrm{P}$. Sijtsma, "Experimental techniques for identification and characterisation of noise sources," in Advances in Aeroacoustics and Applications, VKI Lecture Series 2004-05, edited by J. Anthoine and A. Hirschberg (von Kármán Inst. for Fluid Dynamics, Rhode-Saint-Genèse, Belgium, 2004).

${ }^{18}$ H.-C. Shin, W. R. Graham, P. Sijtsma, C. Andreou, and A. C. Faszer, "Implementation of a phased microphone array in a closed-section wind tunnel," AIAA J. 45, 2897-2909 (2007). 